\title{
Et si les Escherichia coli sélectionnaient les mutations du gène de la mucoviscidose?
}

Les Escherichia coli entérotoxinogènes, ainsi que d'autres bactéries, entraînent des diarrhées aiguës par le biais des entérotoxines. Ces diarrhées sont responsables de $50 \%$ des décès de l'enfant dans les pays en voie de développement. Deux types majeurs d'entérotoxines sont produites par E. coli, une de haut poids moléculaire, thermolabile (LT) et une de petit poids moléculaire, thermostable (ST). Identique à la toxine cholérique, la toxine LT augmente la concentration en AMPc dans les cellules épithéliales de l'intestin grêle par stimulation de l'activité adénylylcylase. La toxine STa, le sous-type le plus commun de toxine ST, stimule, quant à elle, un récepteur spécifique, le STaR, situé de façon prédominante au niveau des membranes apicales des cellules de l'épithélium intestinal. Le gène de STaR a été récemment cloné et s'est avéré être un membre de la famille des récepteurs guanylylcyclase. Un ligand endogène pour STaR vient d'être identifié. Il s'agit d'un peptide de 15 acides aminés, dénommé guanyline, dont l'expression est particulièrement importante dans des cellules cryptiques de la base de l'intestin. Bien que l'on ait su que la toxine STa et la guanyline stimulaient la sécrétion de chlorure par l'intestin en augmentant la concentration de GMPc intracellulaire, le mécanisme par lequel le GMPc modifiait le transport de chlorure restait spéculatif. Une équipe de Stanford (CA, USA) vient de montrer que le GMPc exerce son effet sur la sécrétion de chlorure via la protéine kinase dépendante de l'AMPc (PKA) qui, à son tour, active le canal chlorure CFTR (cystic fibrosis transmembrane conductance regulator) [1]. Ils ont tout d'abord utilisé $\mathrm{m} / \mathrm{s} n^{\circ} 5$ vol. 10, mai 94 la lignée cellulaire T84 dérivée d'un adénocarcinome colique pour mettre en évidence que la toxine STa et la guanyline activaient les courants chlorure. Les effets de la toxine sont mimés par des analogues du GMPc, atténués par des inhibiteurs de la PKA et les courants chlorure observés ont les caractéristiques électrophysiologiques des courants de flux observés à travers le canal chlorure CFTR (T84 exprime fortement (CFTR). Le modèle de fonctionnement normal de la protéine CFTR est le suivant [2]: en l'absence de phosphorylation du domaine $\mathrm{R}$ ( $\mathrm{R}$ : regulatory) de la protéine, domaine central fortement chargé et riche en sérine, le canal est fermé. L'AMPc active la PKA qui phosphoryle un ou plusieurs résidus sérine sur le domaine $R$, ce qui permet à l'ATP de se fixer sur ses deux domaines de fixation. Celui-ci est clivé en ADP et Pi et le changement conformationnel de la protéine CFTR permet le passage des ions selon leur gradient de concentration. Les auteurs présentent trois types d'expériences qui prouvent le rôle du CFTR dans l'action de la toxine STa: (1) le flux sortant entraîné par la toxine est atténué dans des cellules T84 déplétées de CFTR par traitement avec des oligodésoxynucléotides antisens de l'ARN messager du CFTR; (2) un analogue du GMPc augmente le courant chlorure dans les fibroblastes NIH-3T3 seulement après qu'ils ont été transfectés par CFTR (NIH-3T3 est une cellule murine n'exprimant pas (CFTR); (3) cet analogue du GMPc stimule également le courant chlorure de cellules d'épithélium respiratoire dérivées de sujets normaux mais pas de patients atteints de mucoviscidose chez lesquels le gène $C F T R$ est défectueux. Il est intéressant de noter que les études d'hybridation in situ ont localisé la guanyline dans les mêmes cellules que le CFTR, les cellules responsables de la sécrétion de fluide et d'électrolytes, situées dans les cryptes de l'intestin grêle.

La fréquence particulièrement importante de l'allèle muté du $C F T R$ dans la population européenne (1 sur 25) a depuis longtemps fait envisager un avantage sélectif pour les hétérozygotes. La sécrétion de chlorure dépendante de l'AMPc étant défectueuse au niveau du pôle apical des membranes des cellules épithéliales sécrétoires chez les patients atteints de mucoviscidose, il a été proposé que les diarrhées sécrétoires, dues aux toxines de type LT de E. coli qui augmentent la concentration cellulaire d'AMPc, répondaient aux critères de maladie sélectionnant les mutations du CFTR [3, 4]. Les résultats présentés par l'équipe de Chao et al. [1] suggèrent que les toxines dépendantes du GPMc, telles que la toxine STa, pourraient également contribuer à la pression de sélection des allèles mutés du gène CFTR.

E.D.

1. Chao AC, de Sauvage F], Dong Y], Wagner JA, Coeddel DV, Cardner P. Activation of intestinal CFTR $\mathrm{Cl}^{-}$channel by heat-stable enterotoxin and guanylin via cAMPdependent protein kinase. EMBOJ 1994; 13 : 1065-72.

2. Collins FS. Cystic fibrosis : molecular biology and therapeutic implications. Science 1992 ; 256 : 774-9.

3. Hansson G.C. Cystic fibrosis and chloridesecreting diarrhea. Nature 1988; 333 : 711. 4. Baxter PS, Goldhill J, Hardcastle J, Hardcastle PT, Taylor C.J. Accounting for cystic fibrosis. Nature 1988; 335: 211.
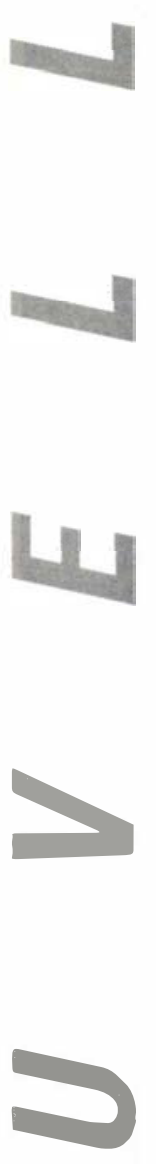